\title{
Gérard Mousseron, 40 années de francophonie à Calgary : Une étude de cas ${ }^{1}$
}

\author{
Gilles Mossière \\ Université Mount Royal
}

Parler de la francophonie dans l'Ouest telle qu'elle se vit au quotidien n'est pas évident, car elle est le fait d'une multiplicité d'origines et de cultures diverses, et l'on sait qu'il est aussi difficile de mettre le doigt sur le présent étant donné que c'est quelque chose qui évolue sans cesse ${ }^{2}$. En effet, philosophes et psychologues le savent bien, le présent est le temps de l'expérience, moment fuyant dans lequel nous baignons et duquel il n'est pas facile de s'extraire pour tenter d'obtenir une vue d'ensemble, une vue surplombante qui pourrait en permettre une compréhension. Pour relever ce défi, notre réflexion sur le sujet s'est donc faite en deux étapes.

Premièrement, nous nous sommes inspiré des deux ouvrages du sociologue Robert Stebbins, professeur émérite de l'université de Calgary: The Franco-Calgarians: French Language, Leisure, and Linguistic Life-Style in an Anglophone City et The French Enigma: Survival and Development in Canada's Francophone Societies, parus respectivement en 1994 et en 2000. Robert Stebbins est un universitaire qui, au cours de sa longue carrière (1976-2000), a publié de nombreux textes sur la sociologie des « loisirs sérieux » (serious leisure), comme il les appelle, dans des domaines divers et assez originaux tels que les monologues comiques (stand-up comedy) ou les quartets de figaros (barbershop singers). Nous espérons montrer que les ouvrages de Stebbins sur la francophonie demeurent des outils d'analyse pertinents.

Un des fils conducteurs de cet article sera la dichotomie entre les notions de "francophonie invisible » - la manière dont les francophones en milieu minoritaire perçoivent leur présence (qui correspond aussi souvent à la perception externe du phénomène francophone en milieu minoritaire) - et de « communauté symbolique »- la perception interne, souvent assez diffuse, que les francophones ont d'eux-mêmes en milieu minoritaire -, notions que nous reprenons de Stebbins (2000, 26-27). Ce dernier a identifié les facteurs suivants

\footnotetext{
1 Version remaniée d'une communication présentée le 28 octobre 2017 au colloque «La présence des francophones européens dans l'Ouest: entre mémoire et actualité » organisé à l'Université de l'Alberta.

2 Les 157 invités des 13 saisons de la défunte émission bilingue de Shaw TV Calgary «Hello/Bonjour Alberta » en ont fourni une preuve indéniable jusqu'en 2017 (ces émissions sont heureusement pérennisées en ligne sur YouTube).
} 
dans la francophonie hors-Québec : 1) le maintien et la transmission de la langue et de la culture, et 2) la croissance et le développement à la fois en termes individuels et communautaires $(1994,11)$. Pour Stebbins, le style de vie des francophones repose sur quatre catégories : la famille, la religion, le travail et les loisirs, à la fois dans le cadre de structures formelles, mais aussi informelles. Peut-être par déformation professionnelle, comme il le reconnaît lui-même, il accorde une grande importance à la sphère des loisirs, qu'il situe à la fois, dans un cadre institutionnel bien défini, mais également au sein de vibrantes sphères communautaires informelles (2000, xi).

Après Stebbins qui fournit le cadre théorico-sociologique de notre propos, pour notre étude de cas nous avons choisi de nous intéresser à Gérard Mousseron. Nous verrons comment ce personnage nous permettra d'illustrer les propos et conclusions de Stebbins, et de relier une expérience individuelle, à la frontière de la fiction et du réel, à celle plus générale de la francophonie albertaine. En effet, Gérard Mousseron est un personnage qui joue un petit rôle dans l'intrigue du roman policier de Laurent Chabin Embrasse ton amour sans lâcher ton couteau, publié au printemps 2016 chez Libre Expression à Montréal. Que ce soit un personnage secondaire - très secondaire même - de ce polar n'enlève rien à notre propos.

Nous dirons d'abord quelques mots sur l'auteur et le roman en question. Laurent Chabin est un auteur assez connu, puisqu'il participe depuis de nombreuses années à divers salons du livre et à des interventions dans des écoles au Québec et dans tout le Canada. Né en France, Chabin a vécu aux Antilles et en Espagne avant de s'installer en Alberta (en juillet 1994), puis quelques années plus tard à Montréal (en février 2007). C'est un auteur prolifique : depuis 1994, il a écrit 84 romans jeunesse et signé 17 romans pour adultes dans le genre du roman policier. Il a aussi publié 9 traductions et collabore actuellement avec Réal Godbout (dessinateur bien connu de la revue satirique Croc dans les années 1970 et 80) sur un scénario de bande dessinée. Si l'on ajoute que certains des auteurs favoris de Chabin sont Céline et Cioran, ce que dit la $4^{\mathrm{e}}$ de couverture d'Embrasse ton amour ne surprendra personne: «ses romans policiers sont noirs, à l'image du monde de violence, de fausseté et de cynisme qu'ils décrivent. » Un certain nombre de ces caractéristiques informent également ses livres pour la jeunesse, ce qui donne une porte d'entrée aux enseignants pour aborder des sujets sensibles avec leurs élèves : pour preuve, la commission scolaire de Saint-John's à Terre-Neuve a commandé 525 exemplaires de 15 ans ferme, roman paru en $2015^{3}$.

${ }^{3}$ Courrier électronique du 16 octobre 2017. 
Quant à Embrasse ton amour sans lâcher ton couteau, c'est le dernier volet de la trilogie consacrée au personnage de Lara Crevier, dont les 2 premiers volumes sont Apportez-moi la tête de Lara Crevier (2014) et Quand j'avais cinq ans, je l'ai tué (2015). La page de garde inclut l'avertissement traditionnel censé dédouaner l'auteur de toute plainte ou poursuite: "Cet ouvrage est une œuvre de fiction; toute ressemblance avec des personnages ou des faits réels n'est que pure coïncidence. » Tout le monde sait ce que cela veut dire. Sans pour autant être un roman à clef, il y a plusieurs serrures assez faciles à forcer dans le polar de Chabin, en particulier celle de Gérard Mousseron.

Venons-en donc à ce personnage, adjuvant très secondaire dans l'intrigue comme nous l'avons dit, puisqu'il n'apparaît qu'entre les pages 162 à 167, dont voici le contexte: pour tenter de retrouver la trace du personnage de Lyne Czemely, le narrateur-détective du roman revient à Calgary pour son enquête et se dirige vers les locaux de l'Alliance française. Il se présente à la directrice sous un faux prétexte pour n'éveiller aucun soupçon. Celle-ci lui dit alors :

- Si vous voulez bien patienter un instant, il y a là quelqu'un qui pourrait vous aider. Gérard Mousseron enseigne ici depuis des années, peut-être a-t-il entendu parler de votre protégée? Son cours est bientôt terminé.

Gérard Mousseron? Ce nom me disait quelque chose. Oui, oui, je connaissais Mousseron. Un peu. L'ennui, c'est qu'il me connaissait aussi... (162)

Le chapitre se termine sur les mots ci-dessus et le début du suivant clarifie la situation :

Évidemment, je ne pouvais pas raconter à Gérard Mousseron les mêmes bobards qu'à sa patronne. Gérard était une figure bien connue de la communauté francophone de Calgary, une figure des plus anciennes et une des rares à parler à tout le monde Québécois, Français, Franco-Albertains, Algériens, Polonais, Vietnamiens, Comoriens et même avec des Ontariens. Il était lié de longue date avec l'Alliance française, mais aussi avec le Centre français de l'université, les radios communautaires et le club de pétanque. (163)

Cette brève introduction permet de situer le personnage: Gérard Mousseron, un Français au nom improbable (peut-être amateur de champignons?), qui ne fait l'objet d'aucune description physique - on n'est pas chez Balzac ni Flaubert ici : c'est un polar, on entre dans le vif du sujet. L'existence de ce personnage semble graviter autour de deux des catégories identifiées par Stebbins : le travail et les loisirs. De sa famille et de sa religion, nous ne saurons rien. En ce qui concerne son travail, nous avons affaire à un enseignant, de classe probablement moyenne puisque l'Alliance française embauche généralement des gens assez cultivés possédant un accent français standard, et qui gravite également autour du milieu universitaire. Cette catégorisation correspond assez bien à celle de la population francophone de 
Calgary que Stebbins a relevée dans son étude dans les années $1990^{4}$ : plutôt de classe moyenne voire supérieure, souvent dans des emplois de cols blancs, de professionnels ou de cadres $(6,1994)$.

Pour les loisirs communautaires, Stebbins fait la distinction entre « passetemps » (casual leisure) et « loisirs sérieux » (serious leisure). Ceux qui s'appliquent ici à Gérard Mousseron sont plus du premier type puisque 1) il n'existe pas de radio communautaire francophone à proprement parler Calgary (on ne saurait considérer Radio-Canada comme de type « communautaire »), et que 2) la météo capricieuse de Calgary ne permet évidemment pas d'envisager la pétanque sous un angle d'activité sportive sérieuse.

Le troisième élément du paragraphe que nous venons de citer tient aux divisions de la communauté francophone de cette ville et à la capacité de Mousseron - rare apparemment, selon le narrateur-détective - de communiquer avec des groupes linguistiques autres que celui de son appartenance d'origine. Il est vrai, et Stebbins l'a bien remarqué, que les activités communautaires à Calgary ont tendance à être fragmentées par «l'ethnicité » et selon « des degrés de différences de culture francophone » : par exemple, les Franco-Canadiens se regroupant autour d'activités particulières; les Nord-Africains de même; et les Français de France, entre autres, autour des activités de l'Alliance française (1994, 27-28; 69-70). On sait d'ailleurs les débats qui animent actuellement l'ACFA et la communauté francophone de l'Alberta pour faire modifier l'appellation «canadienne-française », et la remplacer par quelque chose de plus inclusif. Pour expliquer la capacité de communication de Mousseron - par-delà le côté facétieux de la caractérisation, tel qu'indiqué par la fin de la phrase « et même des Ontariens »-, nous ne pouvons que nous tourner vers l'hypothèse d'une des répondantes du questionnaire de Stebbins selon laquelle «la connaissance d'une autre langue encourage la tolérance » $(1994,104)$. Cela est peut-être vrai, mais la suite du texte apportera peut-être d'autres éléments de réponse plus probants.

Reprenons un peu plus avant dans le chapitre :

$[\ldots]$

[La directrice] a glissé quelques mots à l'oreille [de Mousseron], puis m'a discrètement désigné d'un mouvement du menton. Mousseron m’a dévisagé un moment, sans trahir la moindre émotion, puis il s'est avancé vers le fauteuil où je l'attendais, nonchalamment vautré, jambes croisées, feignant de compter les mouches au plafond.

- Tiens, un revenant.

\footnotetext{
${ }^{4}$ Pour des recherches sur l'immigration plus récente à Calgary, en particulier l'immigration africaine, voir les travaux d'Yvonne Hébert : «Calgary à la lumière : Étude de la redéfinition d'une francophonie urbaine» et "L’immigration de langue française à Calgary » (en collaboration avec Richard Wanner).
} 
Il a assorti cette lapidaire déclaration de bienvenue d'un léger rictus qui pouvait passer pour un sourire. Difficile de savoir ce qu'il avait en tête, sinon, sans doute, un brin de curiosité. Je lui ai proposé d'aller prendre un verre quelque part. Il a hoché la tête et m’a indiqué un bar tout proche qu'il aimait bien, dans la 9e Avenue.

$[\ldots]$

Gérard Mousseron est un homme sympathique, en ceci qu'il ne pose jamais, d'emblée, de question nécessitant une réponse détaillée. "Comment vas -tu? », "Ça faisait longtemps », ou «Quelle surprise! ». Il aime laisser les gens venir à lui. Mais, à ce jeu-là, il n'est pas de taille contre moi. Après une longue giration autour du pot, il a fini par demander, avec un brin d'exaspération :

- Alors comme ça, tu cherches Lyne Czemely?

$[\ldots]$

Mousseron avait connu Lyne quelques années avant moi, alors que, âgée d'une quinzaine d'années, elle était sa plus jeune et sa plus brillante élève à l'Alliance française.

Elle était peu bavarde, mais, ne connaissant sans doute pas encore les grands principes de la discrétion, du mensonge constructif et du brouillage de pistes, elle lui avait raconté qu'elle avait fui sa famille, ne s'entendant pas avec sa mère, et encore moins avec l'homme qui avait plus ou moins remplacé son père après sa disparition.

Autant pour survivre que pour éviter de tomber sous la coupe des services sociaux, dont elle se méfiait comme de la vérole, elle s'était vieillie de quelques années elle faisait de toute façon plus que son âge - et elle avait fini par trouver un protecteur en la personne d'un type assez étrange, Yvan Humbert, de qui elle avait fait connaissance dans un bar fréquenté par des artistes où elle avait pris l'habitude de traîner le soir.

Ce dernier détail, Mousseron le tenait lui-même d'Humbert, vieux beau bien connu des Français de Calgary et qui ne se faisait pas prier pour parler de ses conquêtes, sans préciser pour autant combien ça lui coûtait.

Humbert, un Français expatrié de longue date, assez bobo et plus tout jeune, s'était donc amouraché de notre lolita et avait entrepris de lui faire apprendre sa langue, et bien d'autres choses sans doute. Il l'avait inscrite à l'Alliance française, où Mousseron avait pu apprécier ses talents pour les langues.

$[\ldots]$

De Mousseron, je n'ai rien pu tirer d'autre concernant Lyne ou sa famille. En revanche, certains éléments devaient être publics. Un petit saut s'imposait jusqu'à Edmonton, où je pourrais consulter les archives des journaux locaux.

Il était trop tard pour ce soir. J'ai libéré Mousseron et je suis rentré à l'hôtel ${ }^{5}$.

Dans l'extrait que nous venons de présenter, Mousseron est décrit comme « un homme sympathique», qui «ne pose jamais, d'emblée, de question nécessitant une réponse détaillée », et qui préfère « laisser les gens venir à lui » : en plus de son bilinguisme, on peut supposer que ce sont ces caractéristiques plutôt passives qui mettent les gens en confiance et qui lui permettent d'engager le dialogue avec des groupes linguistiques autres que le sien.

\footnotetext{
${ }^{5}$ Nous avons omis certaines lignes du roman pour ne garder que celles qui font avancer l'intrigue tout en précisant le caractère taciturne de Mousseron, pris dans le « jeu du chat et de la souris » que lui impose le narrateur-détective.
} 
Qui plus est, cet extrait décrit aussi ce personnage en creux, comme par défaut, si l'on peut dire. En effet, la caractérisation de Mousseron est d'autant plus évidente si on la met en rapport avec celle d'Yvan Humbert, personnage totalement imbu de sa personne, un « vieux beau » tirant profit de sa richesse et de son élégance vieille France pour s'attirer les faveurs de jeunes étudiantes impressionnables et en manque d'affection paternelle, comme c'est le cas de Lyne Czemely.

Quant à nous, revenons à l'étude The Franco-Calgarians, livre que Stebbins a dédié à Donna et Henri Mydlarski : Donna, directrice du Centre français de l'université de Calgary de 1979 à 1993, et Henri, professeur de français à cette même université, deux piliers de l'éducation francophone au niveau postsecondaire à Calgary, et qui ont beaucoup aidé Stebbins dans son apprentissage du français et dans ses recherches. Outre Donna et Henri Stebbins remercie également dans sa préface, par ordre alphabétique, Alain Bertrand (qui habitait alors à Calgary, et qui a remporté le $5^{\mathrm{e}}$ tournoi de pétanque avec Michel Cousyn en 2003), Léo Boileau (qui fut notamment président-fondateur du Comité de planification et de la Société du Centre scolaire communautaire de Calgary de 1988 à 1994), Jean-Pierre Deslauriers (professeur de sociologie à l'Université du Québec en Outaouais qui a travaillé avec Stebbins sur plusieurs projets), sa femme Jeanne, Yvonne Hébert (professeure émérite du Département d'éducation à l'université de Calgary après une longue et brillante carrière), ainsi qu'Antoine Sassine (qui a enseigné le français au collège Mount Royal, puis à l'université du même nom, de 1992 à 2015) (1994, xi).

Le nom de Gérard Mousseron, quant à lui, n’apparaît pas dans cette liste. Comme Mousseron était très actif dans le milieu de l'éducation à Calgary, Stebbins lui avait aussi demandé de participer à son étude en 1992, mais Mousseron avait décliné l'invitation. S'il l'avait acceptée, ses commentaires et réflexions auraient été inclus dans les recherches de Stebbins, et les choses en seraient restées là. En effet, pour nous, c'est sa non-participation qui est intéressante et qui va nous permettre, à la fois, d'en savoir plus sur ce personnage et d'apporter un point de vue complémentaire au travail de Stebbins. Mousseron avait décliné de remplir le questionnaire de Stebbins, apparemment parce qu'il n'avait rien à dire sur la francophonie ni la communauté francophone à Calgary. Contrairement aux enseignants et aux personnalités que nous venons de mentionner, Gérard Mousseron n'est donc pas un objet sociologique dans les recherches de Stebbins. En effet, à cette époque-là, les intérêts de Mousseron étaient d'ordre professionnel et financier - enseigner le plus de cours possible, tout en faisant des traductions, afin de subvenir aux besoins de ses enfants - et d'ordre sportif - se maintenir en forme afin de continuer à vivre le plus d'aventures possible en montagne. Son engagement communautaire se limitait 
à une participation à quelques concerts d'artistes francophones, à des films ou des pièces de théâtre. De fait, pour Mousseron, la montagne était ce que Stebbins appelle un " loisir sérieux », - un loisir exigeant en termes de temps et d'énergie -, et ce loisir n'impliquait aucunement la communauté francophone : Mousseron le pratiquait exclusivement avec des partenaires anglophones. Pour lui, cette activité correspondait plus à une recherche personnelle, une recherche d'identité qui ne pouvait se passer que hors de son territoire et de sa communauté linguistique d'origine. Pour Mousseron, l'expérience de la montagne était une découverte de potentialités, ce qui lui a permis au fil des ans de mieux se connaitre et de mieux comprendre sa place dans le monde.
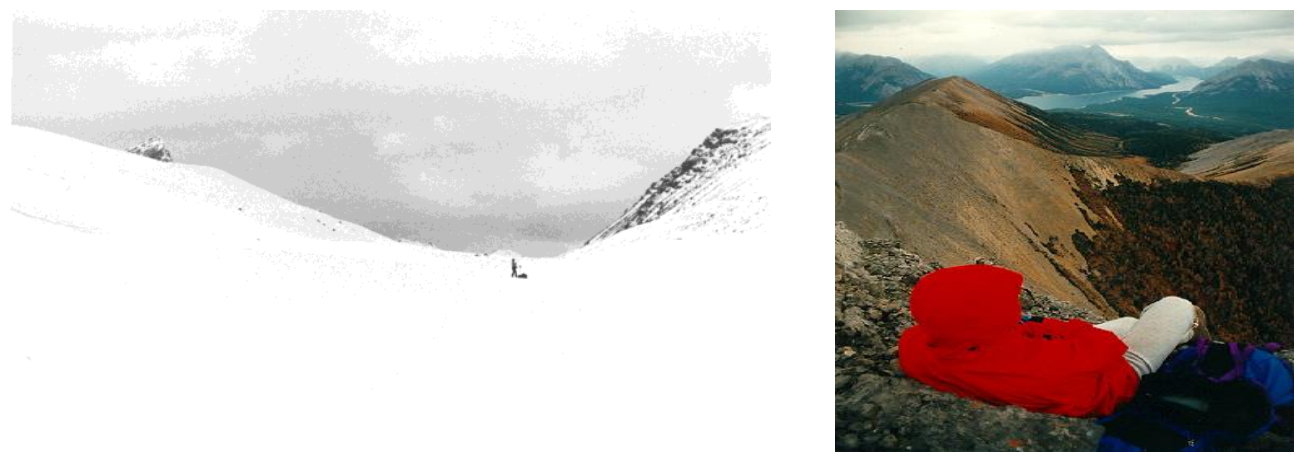

On le voit, par exemple, ici (photo en haut à droite), en direction de Jonas Shoulder, le $2^{\mathrm{e}}$ jour de la traversée hivernale de 4 jours Nigel Pass-Poboktan Creek dans les parcs Banff et Jasper (Shipley), ou encore au sommet du Fist (photo en haut à gauche), regardant l'orage avancer sur les lacs Spray de Kananaskis (Tober). Ce sont les deux seules photos que nous avons de Mousseron. En effet, ce dernier entretient un rapport compliqué avec ce médium qui est censé en dire plus que mille mots : de fait, Mousseron a du mal à se reconnaître dans bien des photos qui sont prises de lui. Est-ce un problème d'estime de soi? Ou quelque chose de plus général qui nous pose problème à tous? Comment réconcilier la vision interne que nous avons de nous-mêmes avec toutes celles que les autres ont de nous, et qu'ils essaient de communiquer en photos (ou par la fiction, dans le cas de Laurent Chabin)? Gérard Mousseron travaillait ce sujet par le biais d'une traduction de l'anglais à laquelle il avait donné le titre "Une histoire de photos », et qu'il a peut-être terminé depuis.

Quoi qu'il en soit, Mousseron avait fait sienne cette citation de René Daumal tirée de Mont Analogue:

On ne peut pas rester toujours sur les sommets. Il faut redescendre...

À quoi bon, alors? Voici : le haut connait le bas, le bas ne connaît pas le haut [...] 
Il y a un art de se diriger dans les basses régions, par le souvenir de ce qu'on a vu, lorsqu'on était plus haut. Quand on ne peut plus voir, on peut du moins encore savoir $(163)^{6}$.

Est-ce un jugement trop prétentieux, qui fait preuve d'un manque flagrant d'humilité, et contraire à l'image de Mousseron que donne Chabin dans son polar? C'est pourtant quelque chose qui a de profonds échos chez tous les montagnards, et qu'il faut sans doute avoir vécu pour le comprendre. Par exemple, comme Horace-Bénédict de Saussure lors de son ascension de l'Etna en Sicile, le 5 juin 1773. Anne Fauche et Samuel Cordier, qui ont réédité le journal de De Saussure en 2007 rappellent que c'est alors qu'il avait pris conscience que «tout apparaît différent depuis un haut sommet » et que cette perception n'avait fait « qu'attiser son désir d'atteindre un jour celui du Mont Blanc », ce qu'il fit 14 ans plus tard (le 3 août 1787), entre autres, pour déterminer la structure et l'origine de la chaîne alpine (36-37) ${ }^{7}$.

De manière étrange, ce détour par la montagne nous ramène une fois de plus à Robert Stebbins. En effet, onze ans après la parution de The FrancoCalgarians et cinq ans après The French Enigma, Stebbins a publié Challenging Mountain Nature : Risk, Motive and Lifestyle in Three Hobbyists Sports, un ouvrage qui décrit et analyse les conditions qui amènent un nombre croissant de gens, comme Gérard Mousseron, à émigrer vers les villes des Rocheuses canadiennes pour y pratiquer des sports de montagne. Dans l'introduction de cette étude, Stebbins explique que lui-même était rapidement «tombé en amour» avec ces montagnes après avoir été embauché à l'université de Calgary en 1976, et qu'il y avait passé beaucoup de temps à faire des randonnées à pied, en raquettes et en ski de fond, ainsi que des ascensions de sommets sans grandes difficultés techniques $(2005,7)$. Si Gérard Mousseron n'est pas un objet sociologique dans The Franco-Calgarians, l'est-il dans Challenging Mountain Nature? La réponse est de nouveau négative. En effet, pour cet ouvrage, Stebbins s'est surtout servi des contacts que sa recherchiste Sherry Kranabitter avait dans la communauté montagnarde de Calgary-Canmore-Banff (11).

\footnotetext{
${ }^{6}$ Puisque Mont Analogue est un roman inachevé, cette citation souvent utilisée ne fait pas stricto sensu du texte rédigé : elle apparaît dans la « Note de l'éditeur » au début d'un " traité d'alpinisme analogique » que Daumal avait écrit avant Mont Analogue, spécifiquement dans la deuxième section intitulée « Définitions », et suit les phrases : «L'alpinisme est l'art de parcourir les montagnes en affrontant les plus grands dangers avec la plus grande prudence. On appelle ici art l'accomplissement d'un savoir dans une action » (162).

${ }^{7}$ Après son ascension du plus haut sommet de l'Europe occidentale, H.-B. de Saussure a écrit une note dans le journal de Genève et rédigé une Relation abrégée d'un voyage à la cime du Mont Blanc en août 1787, mais Le Journal d'un Voyage à Chamouni \& à la cime du Mont-Blanc en juillet et aoust 1787 [sic] n'a été publié pour la première fois qu'en 1926. Voir aussi ses commentaires sur l'orientation, l'architecture et la structure de la chaîne alpine, p. 175-178.
} 
Par contre, la trajectoire montagnarde de Mousseron correspond tout à fait à celle que Stebbins identifie en dix points dans Challenging Mountain Nature, depuis une actualisation et un enrichissement de type tout personnel qui met en jeu l'image de soi dans un premier temps, et qui évolue en un engagement de type social, altruiste, communautaire au fil des années (11). En effet, si Mousseron s'était longtemps tenu à l'écart de la communauté francophone de Calgary - peut-être pour des raisons illustrées de manière caricaturale par le personnage d'Yvan Humbert dans le roman de Chabin -, c'est par la montagne, et par les amitiés qu'il y a forgées pour faire face à ce milieu potentiellement dangereux, qu'il a découvert l'importance de l'appartenance à une communauté. Et l'âge venu, c'est ainsi que Mousseron a fini par s'impliquer en ville dans la communauté francophone de Calgary, et que son engagement communautaire a pris forme, entre autres, en termes de participation radiophonique et de pétanque comme l'écrit Laurent Chabin, mais ce de manière tardive par rapport à l'étude de Stebbins, puisque ces engagements datent respectivement de 1999 et de 2002 .

Nous ajouterons un dernier détail sur le personnage de Gérard Mousseron : son hygiéniste dentaire s'appelle «Désirée Lafleur », et elle serait l'arrière-arrière-petite-fille de Joe «Dollar » Lafleur. Ce nom ne nous disait rien et nous avons dû faire des recherches par Internet pour trouver des détails : Joe «Dollar» La Fleur est un Métis dont le nom est associé au cas de diphtérie qui avait été rapporté en décembre 1928 dans la petite communauté de Little Red River, en aval de Fort Vermilion. Rappelons brièvement les faits. Devant le risque d'épidémie, le médecin avait demandé des bénévoles pour aller de Fort Vermilion à Rivière-La-Paix, une distance d'environ 450 kilomètres, d'où un message télégraphique pourrait être envoyé à Edmonton afin de faire apporter des vaccins dans cette communauté. Le 18 décembre 1928, le Métis Joe «Dollar» La Fleur et son gendre William «Che Chum » Lambert chargèrent donc leurs traîneaux de nourriture et de vêtements pour remonter la rivière. Malheureusement, la glace céda sous le poids du traîneau de Joe et ce dernier tomba dans la rivière. Ils réussirent à s'en sortir et à regagner Fort Vermilion, d'où ils repartirent trois jours plus tard. Par des températures tombant à moins $50{ }^{\circ}$ Celsius, il leur fallut huit jours pour rallier Rivière-La-Paix, le $1^{\text {er }}$ janvier 1929. Ils transmirent leur message d'appel à l'aide et William Lambert fut immédiatement transféré à l'hôpital pour y faire soigner sa pneumonie.

À Edmonton, un sauvetage aérien fut organisé, exploit technique qui eut un retentissement énorme et qui est passé à l'Histoire, mais dans lequel la grande aventure de Joe « Dollar » La Fleur et de William Lambert - $450 \mathrm{~km}$ en traîneau à chiens en 8 jours par des températures polaires - n'a pas été rapportée dans 
les articles publiés à cette époque ${ }^{8}$, ou n'est mentionnée que comme un simple épisode dans les articles consacrés aux aviateurs, en particulier à «Wop » May"

Comme souvent, l'histoire francophone disparaît du devant de la scène, devient «invisible», mais cette histoire demeure vivante au sein de la «communauté symbolique », puisque même son arrière-arrière-petite-fille «Désirée Lafleur» en est fière. Par contre, nous n'avons trouvé aucun autre détail et aucune photo de Joe «Dollar» La Fleur (voir photo ci-dessous).

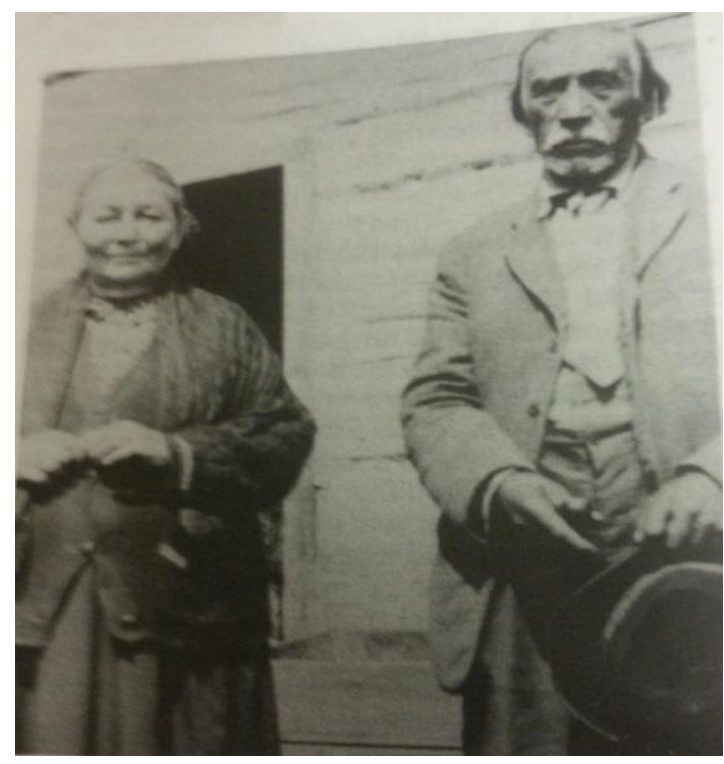

(C)

Comme Laurent Chabin à la recherche de Lyne Czemely, nous nous sommes donc lancé sur la piste de "Désirée Lafleur». Nous l'avons retrouvée, mais son prénom à la consonance bien française s'écrit «Desirae », et elle prononce son nom «Desirae La Fleur» avec un fort accent anglais, car elle ne parle pas un seul mot de français. Le phénomène d'assimilation dont traite longuement Stebbins dans ses livres sur la francophonie a donc joué un rôle déterminant au

${ }^{8}$ Voir les articles du Edmonton Bulletin du 2 au 7 janvier 1929 (en ligne sur le site Our Future Our Past).

${ }^{9}$ On trouve ce résumé dans la première partie de l'article « Mercy Flight of 1929 » dans les Wop May Chronicles (en ligne). Voir aussi l'article en ligne de Beth Wilkins «So-you think we've had it cold - what about... ». Nous ne voudrions pas, à notre tour, minimiser le sauvetage aérien, qui ne fut pas de tout repos non plus, et ces deux articles sont éloquents à ce sujet $-2000 \mathrm{kms}$ allerretour, en 5 jours (dont 2 à Rivière-La-Paix pour réparations), pour un vol total d'environ 14 heures -, mais qui ne mit pas en jeu des efforts humains aussi intenses que l'aventure de Joe La Fleur et de son beau-frère. 
fil des ans (2000, 191-194). En ce qui concerne Desirae, il pourrait que ce soit une assimilation définitive puisque, dans le cadre de ses études, elle s'est rendu compte qu'elle n'avait aucune aptitude pour le français, et qu'elle a depuis épousé un Anglophone unilingue. En tant que francophone, on peut considérer cela déplorable - ce qui n'est nullement son opinion à elle. En tout cas, elle nous a fourni une photo de son arrière-arrière-grand-père et de sa femme Pauline, lors de leurs noces d'or en $1935^{10}$.

Est-ce là donc le sort du présent francophone : l'assimilation à plus ou moins long terme? et le seul souvenir d'une présence symbolique? Stebbins propose des conclusions optimistes par rapport à cet avenir $(2000,194)$ et nous faisons écho à son opinion. La francophonie à Calgary et en Alberta est bien vivante, elle est en renouvellement constant, parfois visible, parfois diffus, souterrain. Mais toutes ces petites histoires individuelles forment un terreau qui permet parfois de les relier à la plus grande histoire, voire à l'Histoire avec un grand H. En effet, comme nous l'avons vu, la quotidienneté francophone dans ce qu'elle a de diffus, voire d'« invisible », finit parfois par donner émergence à un objet littéraire (comme chez Chabin), à des objets sociologiques (Stebbins) ou historiques (Joe «Dollar» La Fleur) qui rendent visible notre communauté

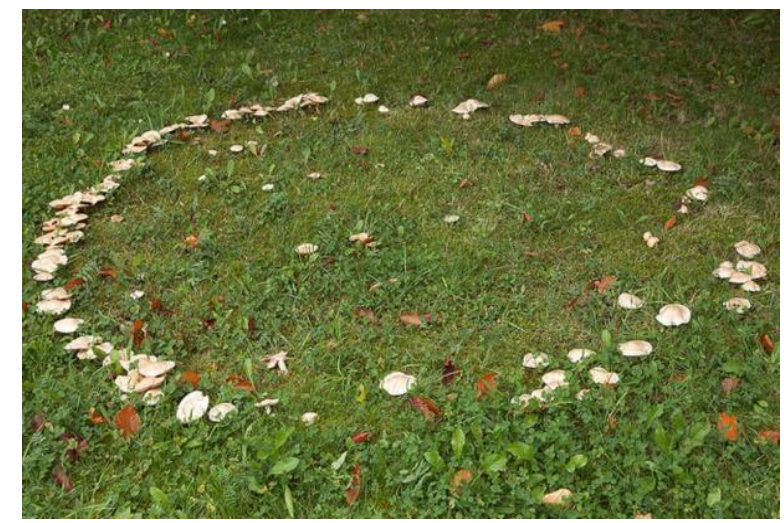

(C)

symbolique. Un peu comme des champignons (voir photo ci-dessus), dont la majeure partie de l'existence se passe en rhizomes souterrains, et qui

\footnotetext{
${ }^{10}$ Desirae ayant mentionné la Société historique de Fort Vermilion, nous avons poursuivi nos recherches et avons retrouvé cette photo dans l'ouvrage publié en 1992 : Fort Vermilion : People in our Vast Trading North (1788-1988), page 24. Ce livre traite aussi de William "Che Chum » Lambert, (37-38) et donne des détails plus précis sur Joe La Fleur (1859-1953 - il était donc âgé de 69 ans quand le cas de diphtérie s'est déclaré) : ayant perdu toutes ses économies parce qu'un gérant de banque indélicat ne les avait pas déposées dans son compte, Joe décida de garder son argent sur lui, ce qui lui valut le surnom de «Dollar. » Son beau-frère et lui furent accueillis en héros à leur retour à Fort Vermilion : Joe reçut une carabine 303 Savage, et William une montre en or, toutes deux gravées à leur nom (24-25).
} 
apparaissent de temps à autre, çà et là, à la surface. Nous trouvons donc que Laurent Chabin a été particulièrement bien inspiré de donner le pseudonyme de «Mousseron » à son personnage, puisque ces champignons poussent en cercles, en communautés, qui rappellent aussi la forme du drapeau de la francophonie internationale ${ }^{11}$.

Nous offrirons également une dernière conclusion optimiste: en tant qu'enseignants, au cours de notre carrière, nous avons l'occasion de toucher des centaines d'étudiants, de les imprégner de la passion que nous avons pour notre langue et notre culture, et qui sait quelles graines nous semons pour l'avenir?

11 Photo personnelle de Gilles Mossière prise en 2017. Qui plus est, les recherches scientifiques indiquent que les champignons jouent un rôle essentiel dans l'écologie des forêts (Semeniuk A3). 


\section{Bibliographie}

Chabin, Laurent. Embrasse ton amour sans lâcher ton couteau, Montréal : Libre Expression, 2016.

Daumal, René. Mont Analogue, édité par H. J. Maxwell et C. Rugafiori, Paris : Gallimard, 1981.

De Saussure, Horace-Bénédict. Journal de l'ascension du Mont-Blanc, édité par Anne Fauche et Samuel Cordier, Chamonix : Éditions Guérin, 2007.

Fort Vermilion and District Bicentennial Association. «La Fleur, Joe Dollar "The Blue Eyed Indian" ", Fort Vermilion: People in our V ast Trading North (1788-1988), p. 24-26.

Hello/Bonjour Alberta.

2017. http://www.youtube.com/playlist?list=PLh9zxWy HXnTZKgM604zli6wo-1HpRvV. Consulté le 15 octobre 2017.

Our Future Our Past. Edmonton Bulletin. 2, 3, 5, 7 janvier 1929. http://www.ourfutureourpast.ca/newspapr/np page2.asp? code $=$ NCXp 0000.jpg. Consulté le15 octobre 2017.

Semeniuk, Ivan, «The real king of the forest is fungus. » The Globe and Mail, 13 janvier 2017, A3.

Shipley, Jim. "Heading towards Jonas Shoulder (Banff-Jasper Park)», Collection personnelle, 1991.

Stebbins, Robert. The Franco-Calgarians: French Language, Leisure, and Linguistic LifeStyle in an Anglophone City, Toronto, University of Toronto Press, 1994.

Stebbins, Robert. The French Enigma: Survival and Development in Canada's Francophone Societies, Calgary, Detselig Entreprises Ltd, 2000.

Stebbins, Robert (2005). Challenging Mountain Nature: Risk, Motive and Lifestyle in Three Hobbyists Sports, Calgary, Detselig Entreprises Ltd, 2005.

The

Wop

May

Chronicles.

2015. [http://wopmay.com/current/adventures/mercyflight.htm. Consulté le 15 octobre 2017. 
Tober, Steve. « On Top of the Fist (Kananaskis)», Collection personnelle. 1993.

Wilkins, Beth. «So - you think we've had it cold - what about...", Peace River Record Gazett. $\quad 9$ janvier 2013. http://www.prrecordgazette.com/2013/01/09/so---you-think-wevehad-it-cold---what-about-. Consulté le 15 octobre 2017. 